\title{
Safety and Risk Associated with Nanoparticles - A Review
}

\author{
Vinita Vishwakarma ${ }^{1 *}$, Subhranshu Sekhar Samal ${ }^{1}$ \& N. Manoharan ${ }^{2}$ \\ ${ }^{1}$ Centre for Nanoscience and Nanotechnology \\ (A Joint Initiative of IGCAR, Kalpakkam \& Sathyabama University, Chennai) \\ ${ }^{2}$ Sathyabama University, Chennai, India-600119 \\ *Corresponding author: vinitavishwakarma1@gmail.com
}

\begin{abstract}
The emerging field of nanotechnology has created risk for environment and human health. Nanoparticles are not a recent discovery. It has existed for many years. Today synthesis of nanoparticles takes place for many applications in various field of science, technology, medicine, colloid technologies, diagnostics, drug delivery, health impacts, food, personal care applications etc. In spite of this, toxicology of nanoparticles is poorly understood as there are no sufficient methods to test nanoparticles for health, safety and environmental impacts, especially in the size range lower than 50nm.
\end{abstract}

Key Words: Nanosafety, nanoparticles, nanotoxicity, health and risk, environmental impact

\section{BACKGROUND}

The behavior of nanoparticles is relatively different from larger particles of the identical material. There is a correlation between a decrease in particle size and an increase in toxicity, because of larger surface area. The ability of nanoparticles to penetrate deep into our respiratory system and their better assimilation in the body fluids make them unique.

\section{NANOPARTICLE PROPERTIES AND SAFETY}

The nanoparticles are likely to be unsafe for the biological system. The research on toxicity of nanoparticles indicates that some of these products may enter the human body and become toxic at the cellular level in the tissues and organs [1,2]. The materials of these particles may or may not be carcinogenic or allergic but even inert nanoparticles show harmful effects due to some absorbed toxic species or formation of toxic products due to reactions with body fluids. Some 
nanoparticles may show enhanced catalytic properties to generate highly reactive forms of oxygen that can cause tissue injury including inflammation and other toxic effects. For air born particles, this injury can translate into asthma and atherosclerotic heart diseases.

The impact of nanoparticles interactions with the body is dependent on their size, chemical composition, surface structure, solubility, shape and how the individual nanoparticles accumulate together. Due to small size and hence higher specific surface area of the nanoparticles, these can easily bind with and transport toxic pollutants, which when inhaled can cause a number of pulmonary diseases in mammals. Inhaled nanoparticles have the ability to translocate in the body as much as $80 \%$ of the deposited mass. Once the nanoparticles enter the body, these can travel freely in the blood throughout the body and reach the organs like liver or brain. It can get deeper into the lungs and bloodstream may cross the blood-brain barrier Skin contact could easily occur during handling of the nanoparticles.

According to the Berkeley proposal, lower sized $(<10 \mathrm{~nm})$ nanoparticles behave more like a gas and can pass through skin and lung tissue to penetrate cell membranes. Once inside the cell, they might become toxic or disrupt normal cell chemistry. Threadlike nanotubes are structurally similar to asbestos fibers, which can cause lung fibrosis when inhaled in large amounts over long periods, according to a report by the Royal Society, the United Kingdom's National Science Academy [3].

Though the nanoparticles are known to exist in the atmosphere in large concentrations $\left(\approx 10^{8} / \mathrm{cc}\right)$, the release of manufactured nanoparticles into atmosphere and aquatic environment is yet insignificant and unknown. But the scientific community has shown concern that nanoparticles cause brain damage in aquatic environment [4].

Fullerenes \& bucky balls, which are known to attract electrons, cause generation of damaging free radicals [5]. Nanotoxicity studies of carbon-based materials as well as quantum dots have been conducted. Literature shows that low-solubility ultrafine particles are more toxic than larger particles on a mass for mass basis [6].

When we consider environmental exposure for nanoparticles, we may find that nanoparticles are easily airborne and adhere easily to surfaces which are difficult to detect. Through the environment nanoparticles may enter the food chain, influence the biosphere, influence structural change in liquids like water (biogenic nanoparticles) and chemical/physical transition by recycling.

According to Nel (2006), some factors like particle size, chemical composition, surface structure, solubility and shape influence the effect of nanoparticles on the body. Such particles can gain access to the body through the gastrointestinal tract, skin and lungs. Nanomaterial may enter inside the body though ingestion. Nel believes that toxicity screening plan for nanomaterials 
should have three key elements - physical and chemical characterization, tissue cellular assays and animal studies.

It is necessary to do more research into the toxicity of nanoparticles because ultrafine particles are more reactive and toxic in their effects. Preliminary studies have shown that some types of nanoparticles could cause lung damage in rats [7].

\section{HANDLING OF NANOPARTICLES}

Some of the organisation like National Institute of Occupational Safety and Health has started an active program for studying the safe handling of nanomaterials in the workplace. How workers are potentially exposed to nanomaterials and if so what are the characteristics and levels of exposures and workers health? What work practices, personal protective equipment, and engineering controls are available and how effective are they for controlling exposures to nanomaterials? [8].

During manufacture and handling of these materials there may be a chance of release and exposure of nanoparticles to workers which can get inside their body through inhalation, dermal contact and ingestion routes [9]. Only limited information on the risks of handling of these materials are available, so workers should implement strict control procedures and engineering safety features to limit exposure when working with them and not to allow them to eat or drink in the laboratory.

When workers handle the nanomaterials they should use laboratory safety practices such as Personnel Protective Equipment (PPE) including gloves, lab coats, safety glasses, face shields, closed-toed shoes etc avoid the skin contact with nanoparticles or nanoparticles containing solutions [10]. If it is necessary to handle nanoparticle powders with exhaust laminar flow hood, workers should wear appropriate respiratory protection. Use of fume exhaust hoods to expel fumes from tube furnaces or chemical reaction vessels is very much crucial. Laboratory personnel should be trained with the risk associated with workplace hazards, Material Safety Data Sheets (MSDS), labeling, signage etc periodically.

Disposal of nanoparticles is also reflecting on the safety of the environment. It should be according to hazardous chemical waste guidelines.

\section{CONCLUSION}

More research is required in this field as ultra fine particles could pose a human health hazard [11]. Research is now showing that when harmless bulk materials are made into ultrafine particles, they tend to become toxic. Generally, the smaller the particles $(<10 \mu \mathrm{m})$, the more reactive and toxic are their effects. 
If nano research helps us to understand the root causes of toxicity in these materials, then safer materials can be engineered which can save to the human lives as well as dollars can be made. Industry consortiums, environmental real data on toxicity into the iterative groups and individual corporations need to take strong action to determine the safety of materials and products before they reach into the market.

Today engineered nanomaterials also help in handling emergencies. Nanotechnology-based sensors and communication devices can reduce their exposure to risk of injury. Nanosize coupled with wireless technology may facilitate development of wearable sensors and systems for real time occupational safety and health management. Nanotechnology-based fuel cells, labon-chip analyzers and opto-electronic devices all have the potential to be useful in the safe, healthy and efficient design. The development of high performance filter media, respirators, dust-repellant, self-cleaning clothes, fillers for noise absorption materials, fire retardants, protective screens for prevention of roof falls and curtains for ventilation control in mines, catalysts for emissions reduction and clean-up of pollutants and hazardous substances may support for nano safety.

Proper care should be taken about nanoparticles and nanotechnology safety issues for the personal health and safety of the workers who are involved in the nanomanufacturing processes and also the consumer to eliminate its effect on the environment.

\section{REFERENCE}

[1] Champeau Rachel., Assessing safety, health risks of Nanomaterials, 2006, published: 15:05 EST, University of California - Los Angeles.

[2] Jin Y., Wu M. and Zhao X., Toxicity of Nanomaterials to Living Cells, University of North Dakota, US, 274 - 277, 2005.

[3] DelVecchio Rick., 2006, Berkeley considering need for Nano safety, articles.sfgate.com.

[4] Rick Weiss, 2004, Nanoparticles Toxic in Aquatic Habitat, Study Finds, Washington Post Staff Writer.

[5] Theresa Phillips., 2009, Are Nanoparticles Safe? About.com Guide.

[6] Cincinnati, OH., Approaches to safe nano-technology: an information exchange with NIOSH,2006a, Department of Health and Human Services, Centers for Disease Control and Prevention, National Institute for Occupational Safety and Health. DHHS (NIOSH), [www.cdc.gov/niosh/topics/nanotech/nano_exchange.html].

[7] Bullis Kevin., 2006, "Nano" Safety Recall: A product touted as "Nano" has hospitalized six German consumers, prompting more warnings over the dangers of Nanomaterials, Technology review.com

[8] Schulte-PA; Salamanca-Buentello-F, Ethical and scientific issues of Nanotechnology in the workplace, Environ Health Perspect 115,5-12, 2007. 
[9] Dreher L. Kevin., 2005, Health and Environmental Impact of Nanotechnology:

Toxicological Assessment of Manufactured Nanoparticles, Toxicological Sciences, 77: 3-5, 2004.

[10] Feder J. Barnaby., Health Concerns in Nanotechnology, 2004, The New York Times.

[11] Peter HM Hoet., Irene Brüske-Hohlfeld and Oleg V Salata, Nanoparticles-known and unknown health risks, Journal of Nanobiotechnology, 2:12 2004. 